QUARTERLY OF APPLIED MATHEMATICS

VOLUME LXVI, NUMBER 2

JUNE 2008, PAGES 233-247

S 0033-569X(08)01124-0

Article electronically published on March 27, 2008

\title{
SENSITIVITY TO NOISE VARIANCE IN A SOCIAL NETWORK DYNAMICS MODEL
}

\author{
BY \\ H. T. BANKS (Center for Research in Scientific Computation, Box 8205, North Carolina State \\ University, Raleigh, North Carolina 27695-8205), \\ A. F. KARR (National Institute of Statistical Sciences, P.O. Box 14006, Research Triangle Park, \\ North Carolina 27709-4006), \\ H. K. NGUYEN (Center for Research in Scientific Computation, Box 8205, North Carolina State \\ University, Raleigh, North Carolina 27695-8205), \\ AND \\ J. R. SAMUELS, JR. (Center for Research in Scientific Computation, Box 8205, North Carolina \\ State University, Raleigh, North Carolina 27695-8205)
}

\begin{abstract}
The dynamics of social networks are modeled with a system of continuous Stochastic Ordinary Differential Equations (SODE). With the proper amount of noise input, the SODE model captures dynamic features that are lacking in the corresponding deterministic ODE model. Therefore, sensitivity to noise levels is investigated by considering four different regimes: essentially deterministic, noise-enriched, noise-enlarged, and noise-dominated. Each regime is defined based on the behavior of solutions of the SODE, and geometry of the regimes are categorized with stochastic simulations.
\end{abstract}

1. Introduction. Social networks are a metaphor and mathematical construct for representing relationships among individuals 1 In a social network, nodes represent individuals, which we term agents, who may have both observable characteristics (for instance, employer or location) and unobservable characteristics (for instance, political or religious affiliation), while edges represent associations between pairs of agents. These associations may be uni-directional - leading to a directed graph, or bi-directional-leading to an undirected graph. Edges may have associated numerical values, representing for example the strength of the association.

Received November 18, 2005.

2000 Mathematics Subject Classification. Primary 91D30, 34F05, 60H10, 60H35, 90B15.

E-mail address: htbanks@ncsu.edu

E-mail address: karr@niss.org

E-mail address: hknguyen@ncsu.edu

E-mail address: jrsamue2@ncsu.edu

${ }^{1}$ Although the metaphor is the same, physical networks such as communication or transportation networks have quite different properties, and are modeled quite differently.

(C)2008 Brown University 
The underlying assumption in most social networks is that pairwise relationships are fundamental. Higher-order relationships are constructed from pairwise ones. Thus, for example, a triad is a set of three agents in which each pair is joined by an edge (i.e., these agents define a complete subgraph). The usual social network framework does not seem to readily accommodate structures such as agents who are members of a committee but have no pairwise relationships.

There is a significant body of research on social networks, within which are threads addressing:

Modeling: often using probability models in which edges occur randomly as a function of the values of observable and unobservable characteristics, which are themselves often random.

Visualization: because some properties of nodes (for example, centrality-having an extremely large number of edges), even though defined mathematically, are strikingly apparent in good visualizations 2

Inference: for example, for parameters of network models.

Prediction: for example of characteristics of the entire network (e.g., average node connectivity) from a sample of the nodes and edges.

Quantitative studies of social networks have been recognized as important for many decades beginning with the work of Moreno [18] in 1934, Katz in 1947 and 1955 [15, 16], and Festinger in 1949 and 1954 [7, 8. Current research can be found in diverse areas including sexually transmitted diseases such as HIV, movie co-starring, needle sharing [12, and terrorist networks [3, 6, to name a few.

There are many types of models used to study social networks including the exponential family of social network models [9] or the $p^{*}$ class of models 26], stochastic blockmodels 23, intelligent agent models [2, 5, 14, 22, and models with latent group structure [1, 13, 19]. For an overview of modern topics and approaches, we refer the reader to [4, 24, 25].

Most of the existing literature deals with static networks, in which neither the set of nodes nor the set of edges varies over time. By contrast, in this paper we introduce a (simple) evolution model of social networks that is motivated by the work of Hoff, et al., [13. We represent the dynamics of relationships between individuals by a coupled system of nonlinear stochastic ordinary differential equations in which the pairwise relationships are driven by both observable and unobservable (latent) characteristics.

In our model we assume that time, the observable characteristics, and individual relationships are continuous. In the spirit of 13 and other models based on concepts of homophily, we assume that an individual's characteristics become more like those of other individuals to which it is linked. We also assume that links between nodes are more likely the higher the similarity of their characteristics. The dynamics (see $\$ 2$ ) are modeled using stochastic ordinary differential equations, in which the randomness represents unobservable factors not captured in the model. An alternative approach to modeling evolution of social network dynamics has been pursued by Snijders [21] using

${ }^{2}$ There is an extensive literature on graph visualization that is largely independent of the social network literature. 
continuous time Markov chain models that are implemented in the software package SIENA (Simulation Investigation for Empirical Network Analysis) [20].

Much of our attention focuses on qualitative behavior of the model as the noise parameters associated with stochastic disturbances vary. Heuristically, as the variance terms in the stochastic differential equations increase from 0 , the model at first differs little from the deterministic case (in which they are 0). For somewhat larger values, the model enters a noise-enriched regime, in which its behavior is richer, but its ultimate fate remains that of the deterministic case. For still larger values of the variances, there may be noise-enlarged behavior: a larger set of long-term behaviors is possible. Finally, for sufficiently large noise variances, the model is noise-dominated, with no structure. Of course, the boundaries between these regimes are not sharp, and so we devote some attention simply to defining them. Nor is it clear that all exist in any specific situation.

2. Mathematical model. We denote by $\mathbf{C}_{i}(t) \in K \subset \mathbb{R}^{m}$ the vector of characteristics of agent $i, i \in \mathcal{N}=\{1, \ldots, N\}$ at time $t$, where $m$ is the number of characteristics and $K$ is a (compact) constraint set for the values of the characteristics.

Let $e\left(i, i^{\prime}, t\right) \in \mathbb{R}$ be the degree of connectivity between agent $i$ and agent $i^{\prime}$ at time $t$. Then a link or edge is present from agent $i$ to agent $i^{\prime}$ at time $t$ if (and only if) $e\left(i, i^{\prime}, t\right)>0$. By convention there are no "self links" so that $e(i, i, t)=0$ for all $i$ and $t$. We do not require that connectivity be symmetric, so it is possible that $e\left(i, i^{\prime}, t\right) \neq e\left(i^{\prime}, i, t\right)$.

We let

$$
A_{i}(t)=\left\{i^{\prime} \in \mathcal{N}: e\left(i, i^{\prime}, t\right)>0\right\}
$$

represent the set of all agents to which agent $i$ has links at time $t$ and let $\left|A_{i}(t)\right|$ be the number of elements in $A_{i}(t)$. Then the basic stochastic differential equations in our model are

$$
\begin{aligned}
d \mathbf{C}_{i}(t) & =\frac{\beta_{i}}{\left|A_{i}(t)\right|} \sum_{i^{\prime} \in A_{i}(t)}\left[\mathbf{C}_{i^{\prime}}(t)-\mathbf{C}_{i}(t)\right] d t+\sigma d \mathbf{W}_{i}^{\mathbf{C}}(t) \\
d e\left(i, i^{\prime}, t\right) & =f\left(\left\|\mathbf{C}_{i}-\mathbf{C}_{i^{\prime}}\right\|^{2}\right) d t+\gamma d W_{i, i^{\prime}}^{e}(t) .
\end{aligned}
$$

The coupling between (2.1) and (2.2) is via $A_{i}(t)$ since $A_{i}(t)$ depends on the vector $\mathbf{e}_{i}(t)=\left(e(i, 1, t), \ldots, e\left(i, i^{\prime}-1, t\right), e\left(i, i^{\prime}+1, t\right), \ldots, e(i, N, t)\right)$. In (2.1),$\beta_{i}>0$ is an agent-specific factor that can be interpreted as the degree to which agent $i$ seeks to become more like other agents to which it is linked, and $\sigma \mathbf{W}_{i}^{\mathbf{C}}(\cdot)$ is a (vector-valued) Wiener process with variance $\sigma^{2}$. In (2.2), $f(\xi)=2 e^{-b \xi}-1$, where $b>0,\|\cdot\|$ denotes the norm in $\mathbb{R}^{m}$, and $\gamma W_{i, i^{\prime}}^{e}(\cdot)$ is a Wiener process with variance $\gamma^{2}$.

Note that (2.1) actually consists of $N m$ equations, while (2.2) consists of $N(N-1)$ equations. These are to be solved subject to the state constraint $\mathbf{C}_{i}(t) \in K$, with given initial conditions $\mathbf{C}_{i}(0)$ and $\mathbf{e}_{i}(0)$. All $N m+N(N-1)$ Wiener processes are assumed independent.

The interpretation of these equations is as described informally in $\$ 1$ First, (2.1) stipulates that the characteristics of agent $i$ move at rate $\beta_{i}$ toward the centroid of the characteristics of the agents to which $i$ has links, subject to random perturbation from $\sigma \mathbf{W}_{i}^{\mathbf{C}}(\cdot)$. Then (2.1) states that the degree of connectivity between $i$ and $i^{\prime}$ increases or decreases at rate $f\left(\left\|\mathbf{C}_{i}-\mathbf{C}_{i^{\prime}}\right\|^{2}\right)$, again with a Wiener-driven perturbation. 
The particular function $f$ in (2.2) is somewhat arbitrary. The important properties are that $f=1$ when characteristics are identical, causing connectivity to increase at rate 1 , and $f<0$ when two agent's characteristics are sufficiently unlike, causing their connectivity to decrease. The value for which $f=0$, and hence characteristics have no effect on connectivity, depends on $b$. The impact of $b$ on formation of links is discussed in 4.1

We note that (2.1) does not satisfy typical assumptions regarding smoothness of coefficients; an alternative, smoother model is described in $\$ 5$.

3. Numerical method. In solving the above SODEs (2.1) and (2.2) subject to the constraints $\mathbf{C}_{i}(t) \in K$, we use the stochastic analogue of the classical fourth-order RungeKutta method [10. Although an implicit numerical scheme [17] would have more stability, we successfully used a familiar and simpler scheme to demonstrate model properties in our initial calculations. Before we write down the algorithms of the fourth-order Runge-Kutta method, we rewrite equation (2.1) as follows:

$$
d \mathbf{C}_{i}(t)=\mathbf{g}\left(t, \mathbf{C}_{i}(t)\right) d t+\sigma d \mathbf{W}_{i}^{\mathbf{C}}(t),
$$

where $\mathbf{g}\left(t, \mathbf{C}_{i}(t)\right)=\frac{\beta_{i}}{\left|A_{i}(t)\right|} \sum_{i^{\prime} \in A_{i}(t)}\left[\mathbf{C}_{i^{\prime}}(t)-\mathbf{C}_{i}(t)\right]$. We partition the interval $\left[t_{0}, T\right]$ uniformly by the step size $h=\Delta t_{n}=t_{n}-t_{n-1}$. Then we can approximate $\mathbf{C}_{i}\left(t_{n}\right)$ by the following:

$$
\mathbf{C}_{i}\left(t_{n}\right)=\mathbf{C}_{i}\left(t_{n-1}\right)+p_{0} \mathbf{F}_{0} h+p_{1} \mathbf{F}_{1} h+p_{2} \mathbf{F}_{2} h+p_{3} \mathbf{F}_{3} h+\sigma \Delta \mathbf{W}_{i n}^{\mathbf{C}},
$$

where

$$
\begin{aligned}
\mathbf{F}_{0} & =\mathbf{g}\left(t_{n-1}, \mathbf{C}_{i}\left(t_{n-1}\right)\right) \\
\mathbf{C}_{i}^{(1)}\left(t_{n-1}\right) & =\mathbf{C}_{i}\left(t_{n-1}\right)+\frac{1}{2} \mathbf{F}_{0} h+\frac{1}{2} \Delta \mathbf{W}_{i n}^{\mathbf{C}} \\
\mathbf{F}_{1} & =\mathbf{g}\left(t_{n-1}+\frac{1}{2} h, \mathbf{C}_{i}^{(1)}\left(t_{n-1}\right)\right) \\
\mathbf{C}_{i}^{(2)}\left(t_{n-1}\right) & =\mathbf{C}_{i}\left(t_{n-1}\right)+\frac{1}{2} \mathbf{F}_{1} h+\frac{1}{2} \Delta \mathbf{W}_{i n}^{\mathbf{C}} \\
\mathbf{F}_{2} & =\mathbf{g}\left(t_{n-1}+\frac{1}{2} h, \mathbf{C}_{i}^{(2)}\left(t_{n-1}\right)\right) \\
\mathbf{C}_{i}^{(3)}\left(t_{n-1}\right) & =\mathbf{C}_{i}\left(t_{n-1}\right)+\mathbf{F}_{2} h+\Delta \mathbf{W}_{i n}^{\mathbf{C}} \\
\mathbf{F}_{3} & =\mathbf{g}\left(t_{n-1} h, \mathbf{C}_{i}^{(3)}\left(t_{n-1}\right)\right), \\
p_{0}=p_{3} & =\frac{1}{6} \text { and } p_{1}=p_{2}=\frac{1}{3} .
\end{aligned}
$$

Here $\Delta \mathbf{W}_{i n}^{\mathbf{C}}=\mathbf{W}_{i}^{\mathbf{C}}\left(t_{n}\right)-\mathbf{W}_{i}^{\mathbf{C}}\left(t_{n-1}\right)$ are standard Wiener increments ([1], p. 489), which are obtained as sample values of normal random variables with the mean of zero and variance $\Delta t_{n}$. Equation (2.2) can be approximated in a similar manner, except it is simpler than equation (2.1) since the right-hand side of equation (2.2) is independent of the degree of connectivity $e\left(i, i^{\prime}, t\right)$.

The discretized system is solved subject to the constraint $\mathbf{C}_{i}\left(t_{n}\right) \in K$. For any step resulting in a value violating this constraint, the value is replaced by the nearest value on the boundary of $K$. 
4. Numerical simulations. To illustrate the concepts introduced in $\$ 1$ we employ an example with ten agents $(N=10)$ and two characteristics $(m=2)$ for each agent, each constrained to lie in $[-10,10]$. Thus $K=[-10,10] \times[-10,10]$. For concreteness, we term the first characteristic $C_{i}^{\mathrm{sq}}$ a "sociability quotient" (positive values represent higher sociability) and the second an "outlook on life" denoted by $C_{i}^{\text {out }}$. For the latter, -10 is construed as a "negative outlook" and 10 a "positive outlook." For simplicity, we set $\beta_{i}=1$ for all $i$, which in effect simply calibrates the time scale.

The initial values of the characteristics are set as $\mathbf{C}_{2}(0)=(-10,-10), \mathbf{C}_{7}(0)=$ $(-5,-5)$, and $\mathbf{C}_{i}(0)=(10,10)$ for $i \neq 2,7$. We define the initial conditions for the connectivities $e$ by $\mathbf{e}_{i}(0)=\mathbf{0}$.

4.1. Deterministic case. In the deterministic case that $\gamma=\sigma=0$, the behavior of the model depends (only) on the value of $b \in(0, \infty)$. For $b \in(0.105, \infty)$, neither agent A2, the "extreme outlier," nor agent A7, the "moderate outlier," forms edges with any other agents. The remaining agents, of course, form edges and their connectivity continues to increase. This behavior can be seen by examining the degrees of connectivity $e\left(2, i^{\prime}, T\right), e\left(7, i^{\prime}, T\right)$, and $e\left(10, i^{\prime}, T\right)$ (throughout, we use A10 as an exemplar of all agents other than A2 and A7) at $T=10$. We illustrate in Figures 1, 2, and 3 this three-cluster scenario. The plots satisfy $e\left(2, i^{\prime}, T\right)<0, e\left(7, i^{\prime}, T\right)<0$ for all $i^{\prime}$, and $e\left(10, i^{\prime}, T\right)<0$ for $i^{\prime}=2,7$, while $e\left(10, i^{\prime}, T\right)>0$ for $i^{\prime} \neq 2,7$.

Specifically, in Figure 1 the connectivity between A2 and the other nine agents is negative at $T=10$. In other words, A2 does not have links to any other agent at the final time. We see the same result for A7 in Figure 2. Finally, Figure 3 shows that A10 has no links (negative connectivity) with A2 and A7, but links (positive connectivity) to all other agents.

A two-cluster regime arises for $b \in[0.033,0.105]$. One cluster contains A2 and A7, and the other cluster consists of the remaining agents. As we illustrate in Figure 4, A2 becomes linked to A7 by $T=10$, but not to any other agents. Figure 5 shows that A7 is linked to A2 but not to any of the remaining agents. Figure 6 shows that A10 has links to all agents except $\mathrm{A} 2$ and $\mathrm{A} 7$.

Finally, for $b \in(0,0.033)$, we have a one-cluster case: all ten agents become linked to one another. By observing the connectivity of A2 in Figure 7, A7 in Figure 8, and A10 in Figure 9, we note that A7 is more strongly connected to the group than A2 (as expected since A7 is the "moderate outlier"). Moreover, A7 and A2 have higher connectivity to one another than with the remaining agents. 


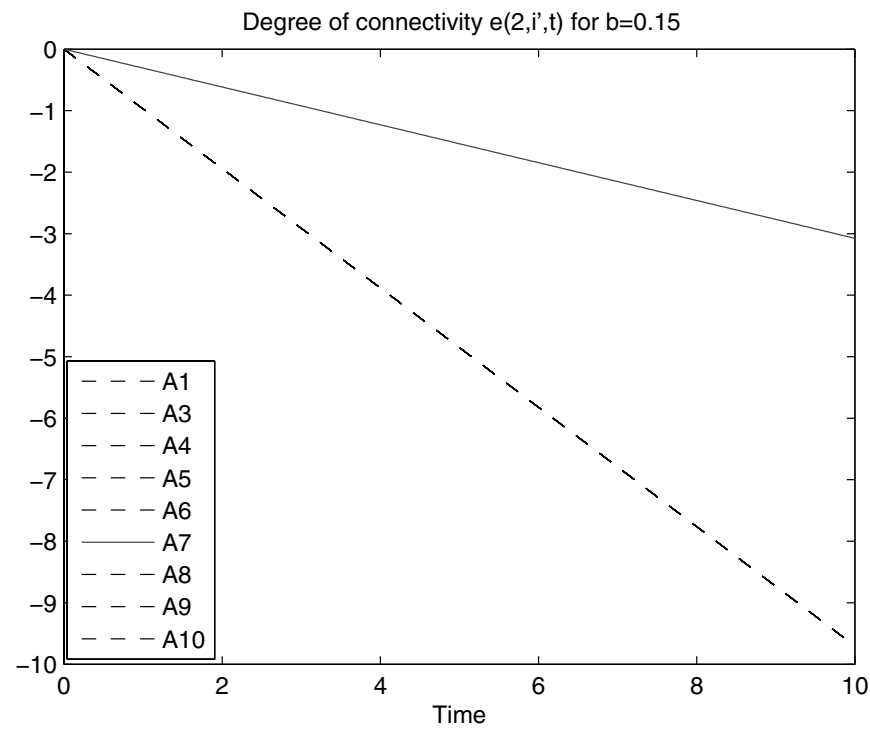

FIG. 1. Degree of connectivity $e\left(2, i^{\prime}, t\right)$ of agent 2 (the "extreme outlier") with other agents for $b=0.15$. Note that the curves for $i^{\prime}=1,3-6,8-10$ all lie on the same line in this 3 -cluster scenario.

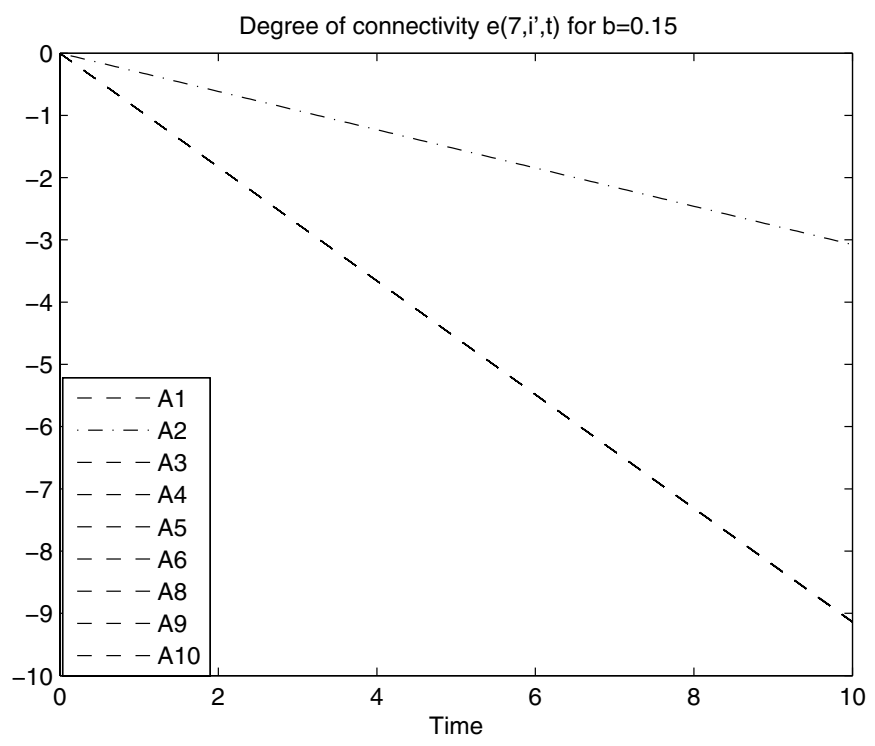

FIG. 2. Degree of connectivity $e\left(7, i^{\prime}, t\right)$ of agent 7 (the "moderate outlier") with other agents for $b=0.15$ (again the 3cluster scenario). 


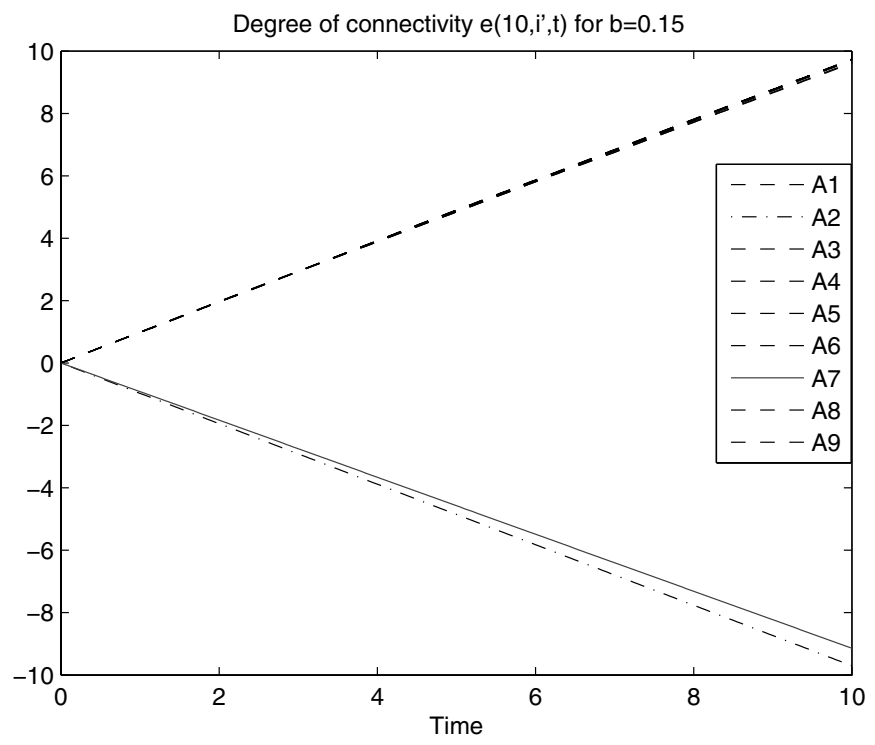

FIG. 3. Degree of connectivity $e\left(10, i^{\prime}, t\right)$ of agent 10 with other agents (except for 2 and 7 ) for $b=$ 0.15 (again a 3 -cluster scenario).

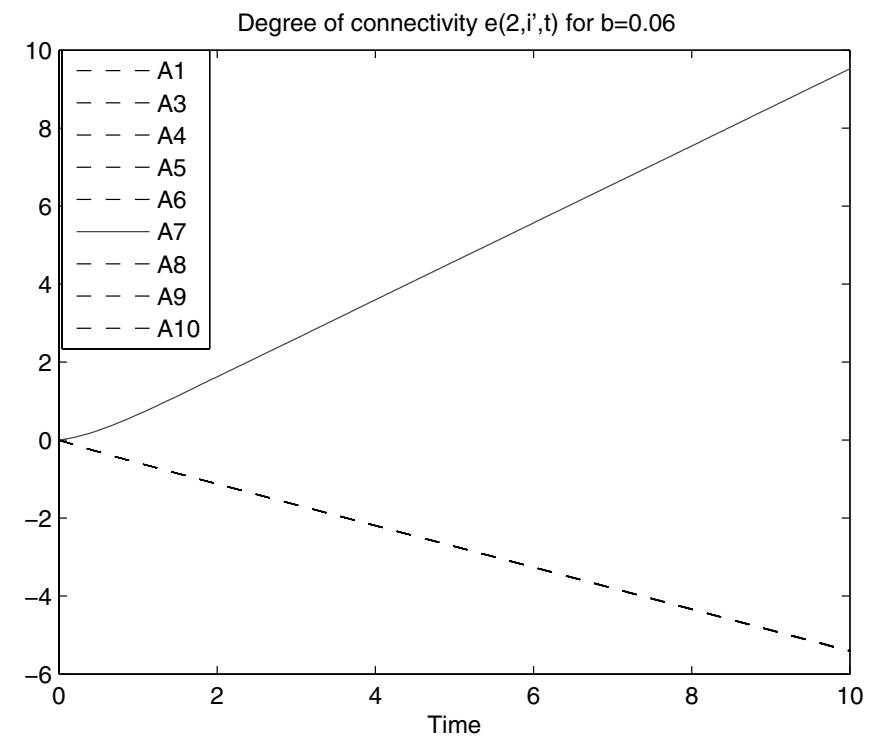

FIG. 4. Degree of connectivity $e\left(2, i^{\prime}, t\right)$

of agent 2 with other agents for $b=0.06$ illustrating the two-cluster scenario. 


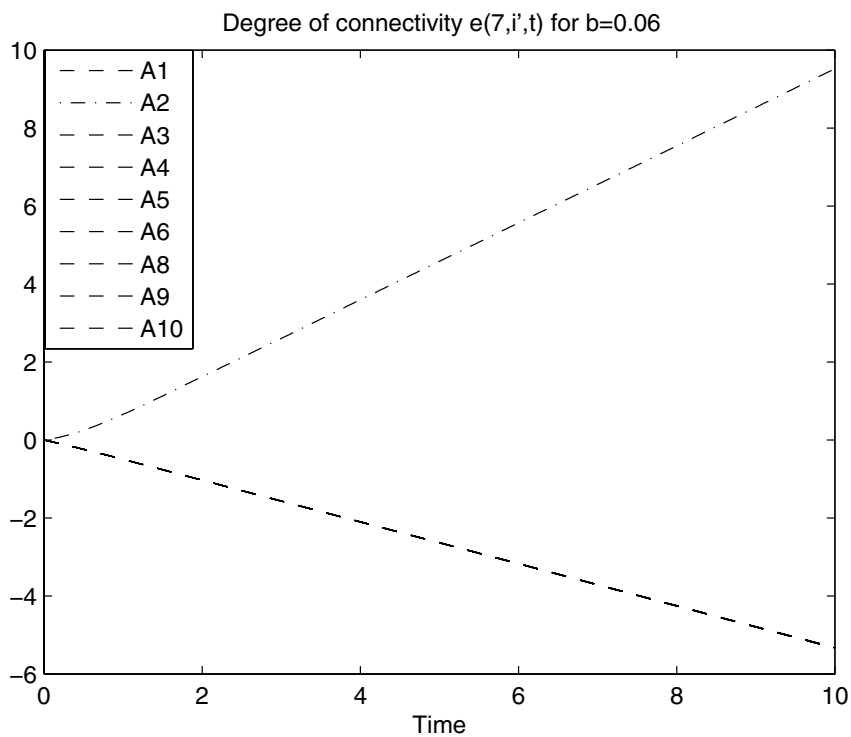

FIG. 5. Degree of connectivity $e\left(7, i^{\prime}, t\right)$ of agent 7 with other agents for $b=0.06$ (again the 2-cluster scenario).

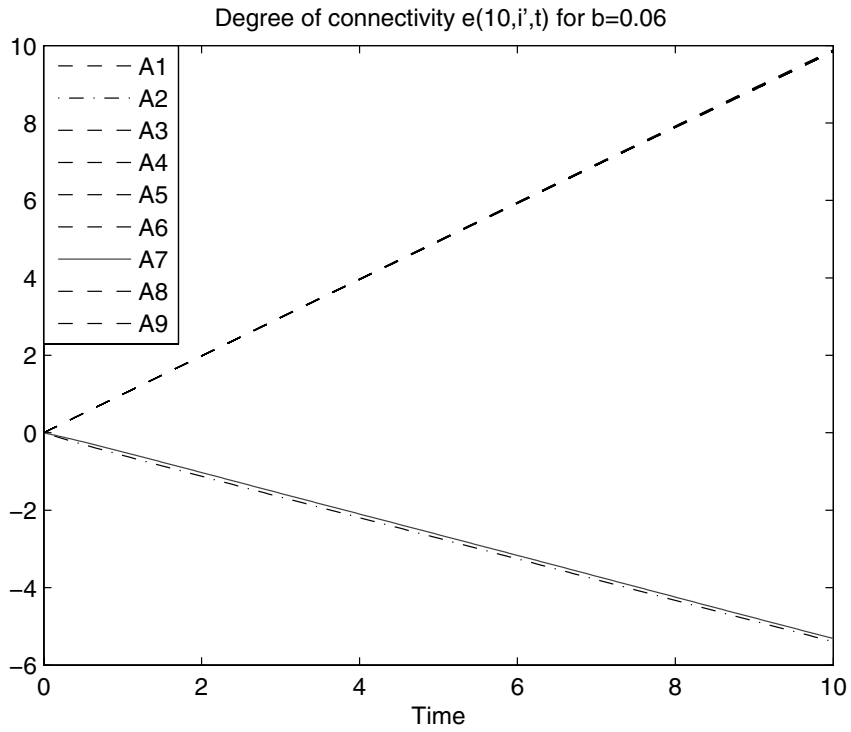

FIG. 6. Degree of connectivity $e\left(10, i^{\prime}, t\right)$ of agent 10 with other agents for $b=0.06$ (2-cluster scenario). 


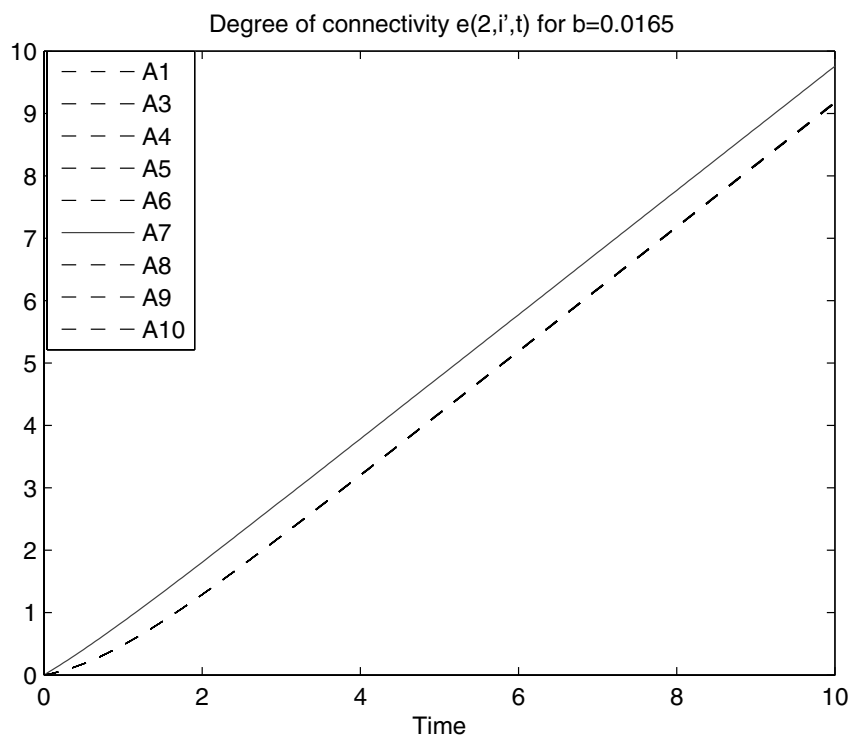

FIG. 7. Degree of connectivity $e\left(2, i^{\prime}, t\right)$ of agent 2 with other agents for $b=0.0165$ illustrating the single-cluster scenario.

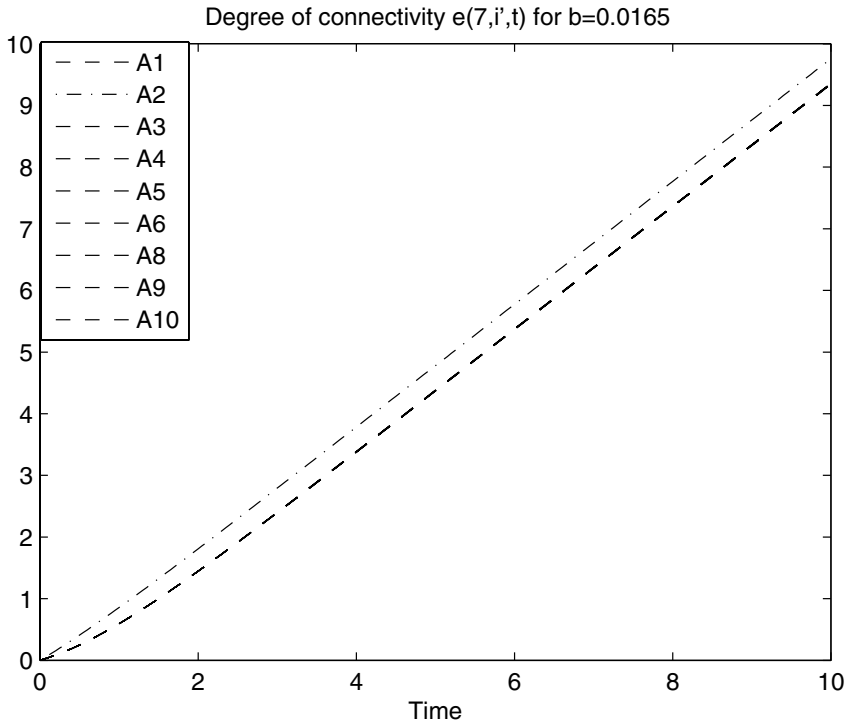

FIG. 8. Degree of connectivity $e\left(7, i^{\prime}, t\right)$ of agent 7 with other agents for $b=0.0165$ (single-cluster scenario). 


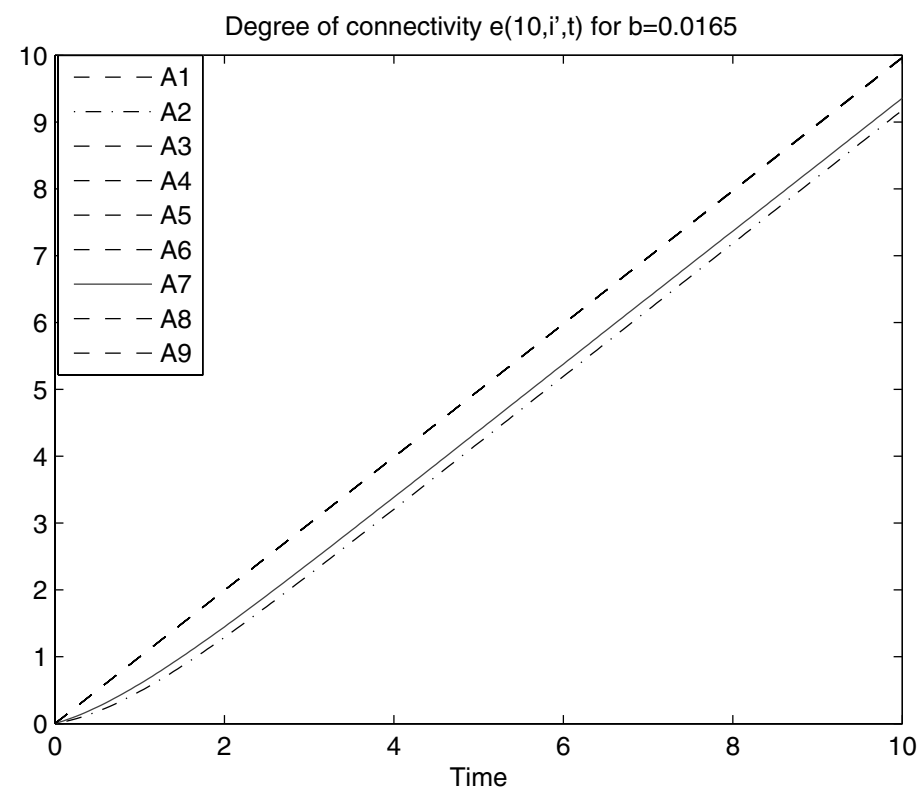

FIG. 9. Degree of connectivity $e\left(10, i^{\prime}, t\right)$ of agent 10 with other agents for $b=$ 0.0165 (single-cluster scenario).

4.2. Stochastic case: Regime definition. We categorize pairs $(\sigma, \gamma)$ based on the behavior of solutions of the SODEs (2.1) and (2.2) with fixed $b=0.0165$. Recall from 44.1 that in this case the deterministic system leads to a single cluster, where A7 has a higher connectivity with other agents than A2, and A7 and A2 are more connected to one another than to other agents for all time $t$.

With the stochastic terms included (that is, $\gamma>0$ and $\sigma>0$ ), we expect a broader range of behavior for the solutions to (2.1) and (2.2). In \$1, we defined four regimes heuristically. For the example, we formalize those definitions as follows. The final time is $T=30$.

Essentially deterministic: consists of $(\sigma, \gamma)$ pairs for which all of the following are satisfied:

- ED1: For all $t \leq T$, all connectivities between all agents are nonnegative: $e\left(i, i^{\prime}, t\right) \geq 0,0 \leq t \leq T, i \neq i^{\prime}$.

- ED2: For all $t \leq T, \mathrm{~A} 7$ and A2 have higher connectivity with each other than the rest of the group: $e(2,7, t)>e\left(2, i^{\prime}, t\right)$ for $i^{\prime} \neq 7$ and $e(7,2, t)>e\left(7, i^{\prime}, t\right)$ for $i^{\prime} \neq 2$.

- ED3: For all $t \leq T, \mathrm{~A} 7$ has higher connectivity with others than does A2: $e(i, 7, t)>e(i, 2, t)$ for $i \neq 2,7$.

- ED4: At $t=T$, all agents have links to one another: $e\left(i, i^{\prime}, T\right)>0$ for $i \neq i^{\prime}$. That is, in the essentially deterministic regime, all qualitative aspects of the deterministic case are preserved. 
Noise-enriched: consists of $(\sigma, \gamma)$ pairs for which any of ED1-ED3 fails, but ED4 holds. That is, the agents take different paths to reach the final destination of being linked to one another at $T$, so that the social network evolves to a single cluster.

Noise-enlarged: consists of $(\sigma, \gamma)$ pairs for which ED4 also fails, but either the three-cluster or two-cluster "equilibrium" in $\$ 4.1$ arises. Put differently, $(\sigma, \gamma)$ is in the noise-enlarged regime if either of the following holds:

- NE1: At time T, A7 and A2 are linked in one group and the remaining agents are linked in another group (two clusters).

- NE2: At time T, A7 and A2 are not linked to any other agents, but all agents other than A2 and A7 are linked to one another (three clusters).

Noise dominated: consists of $(\sigma, \gamma)$ pairs for which none of the three structured outcomes described above obtains.

To investigate whether these regimes exist and, if so, to characterize their properties, we ran simulations, with time step $h=\Delta t_{n}=0.05$, for selected values of $\sigma \in[0,2.7]$ and $\gamma \in[0, .8]$. We carried out 100 simulations for each pair $\left(\sigma_{k}, \gamma_{l}\right)$ in a partition with values $\sigma_{k}=.1 k, k=1, \ldots, 27, \gamma_{l}=.01 l, l=1, \ldots, 80$.

We depict the surfaces representing the noise-enriched and noise-dominated regimes in Figures 10 and 11, respectively. The noise-dominated surface is almost the complement of the noise-enriched surface. A few cases of essentially deterministic behavior are seen at small values of $\sigma$ and $\gamma$.

Therefore, we used the refined partition $\sigma_{k}=.01 k, k=1, \ldots, 70, \gamma_{l}=.001 l, l=$ $1, \ldots, 50$ to attempt to delineate the essentially deterministic regime. The corresponding essentially deterministic and noise-enriched surfaces are plotted in Figures 12 and 13 , respectively. These two plots are almost complements of one another because there are few cases of noise domination.

5. Concluding remarks. We observe from the simulations reported in 4.2 that regimes appear to be more sensitive to $\gamma$ than to $\sigma$. This may be because $\gamma$ directly affects edge formation, which is critical in regime definition, whereas $\sigma$ does not. We suspect that if the regime definitions were based upon the observed characteristics, which are directly influenced by $\sigma$, then the sensitivities observed might be reversed.

There is no evidence in our simulations for existence of a noise-enlarged regime. We believe this is because the criteria that define the noise-enlarged state depend on a nonlinear term involving $b$, whereas the randomness $\sigma$ and $\gamma$ enter the dynamics linearly. In order to verify this claim, we would need to pursue a rigorous sensitivity analysis for the SODEs (2.1) and (2.2).

Although we have a simple model, the richness and flexibility of the model are substantial due to the model parameters. For example, we can significantly enrich the model by allowing the parameter $b$ in the connectivity formation function $f$ to be agent-dependent. Moreover, we might have $\beta_{i} \in \mathbb{R}$ instead of $\beta_{i}>0$ to describe situations where agent $i$ is either repelled by or attracted to the characteristics of his acquaintances. We would then expect the model to exhibit more than just the three regime cases described above. Since exhaustive simulation studies are not particularly desirable (nor feasible as the 


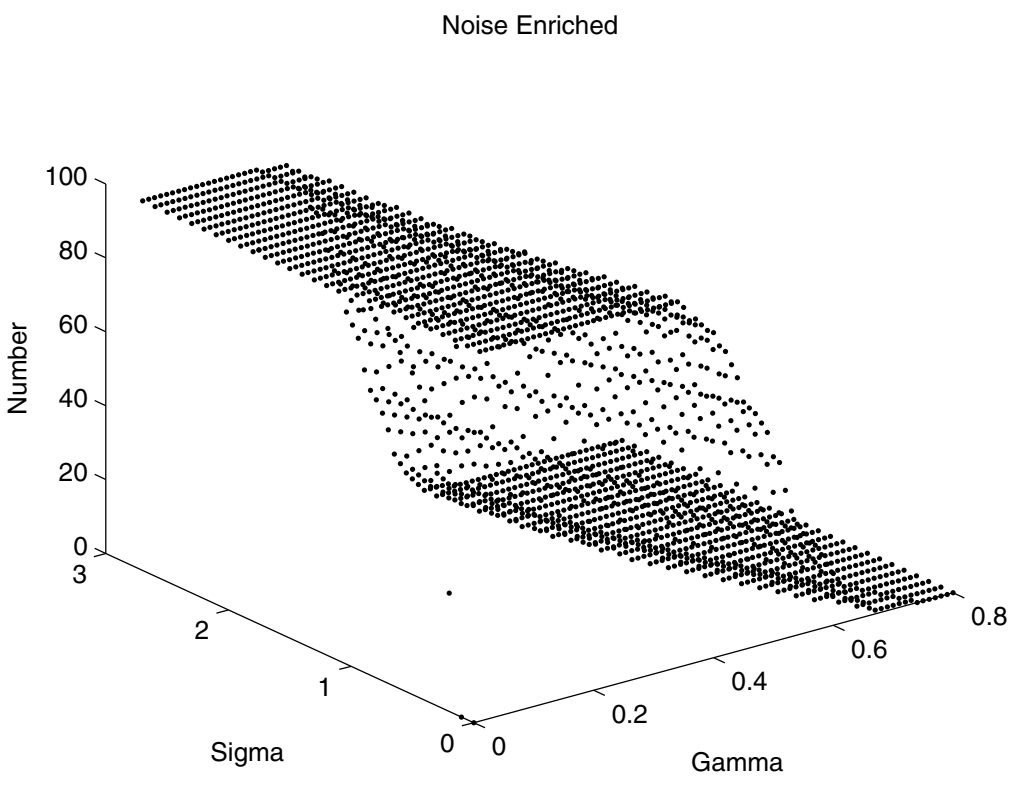

Fig. 10. The $(\sigma, \gamma)$ noise-enriched surface plotted as a percentage or number out of 100 simulations.

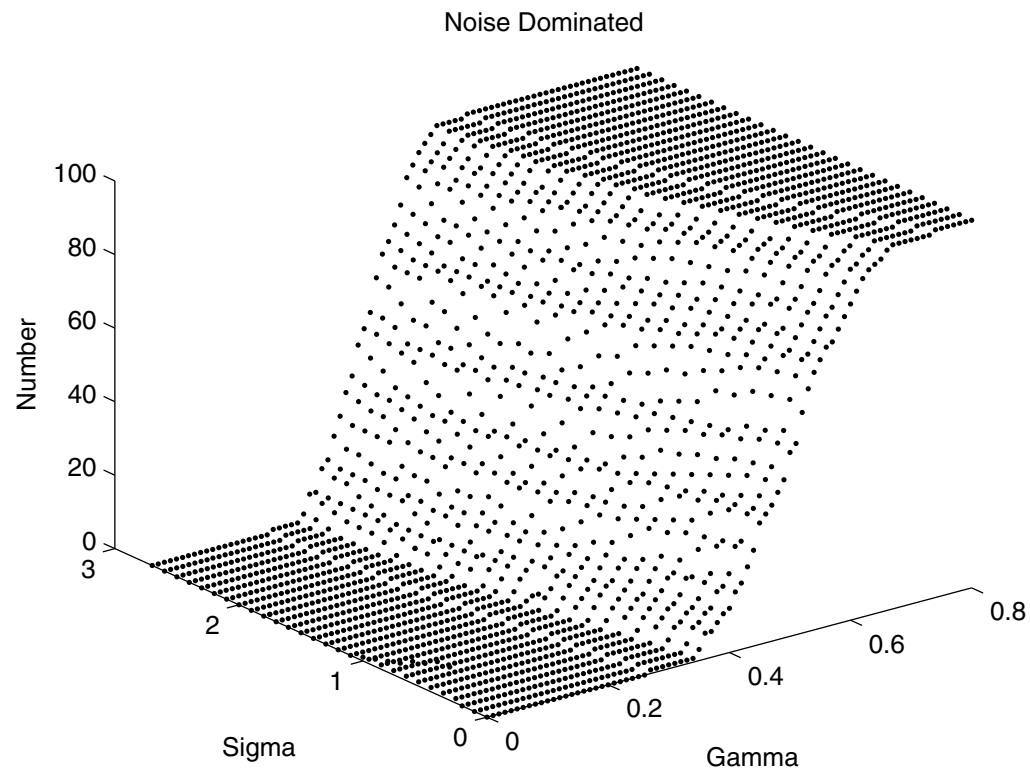

FIG. 11. The $(\sigma, \gamma)$ noise-dominated surface. 


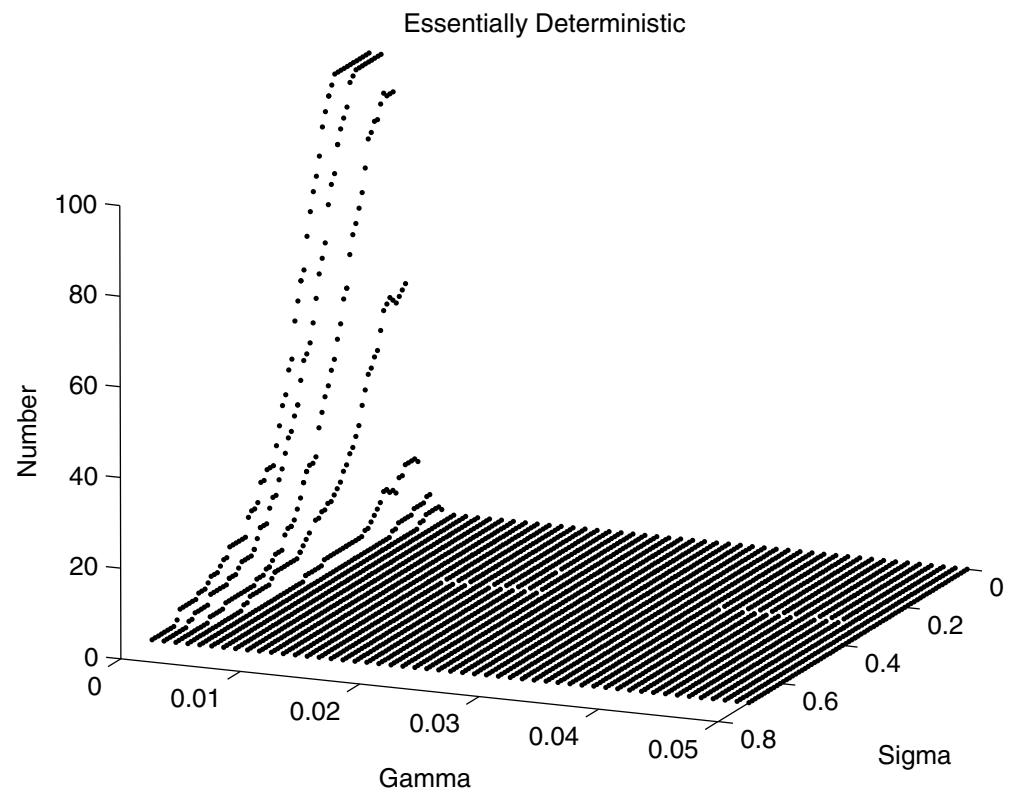

FIG. 12. The $(\sigma, \gamma)$ essentially deterministic surface.

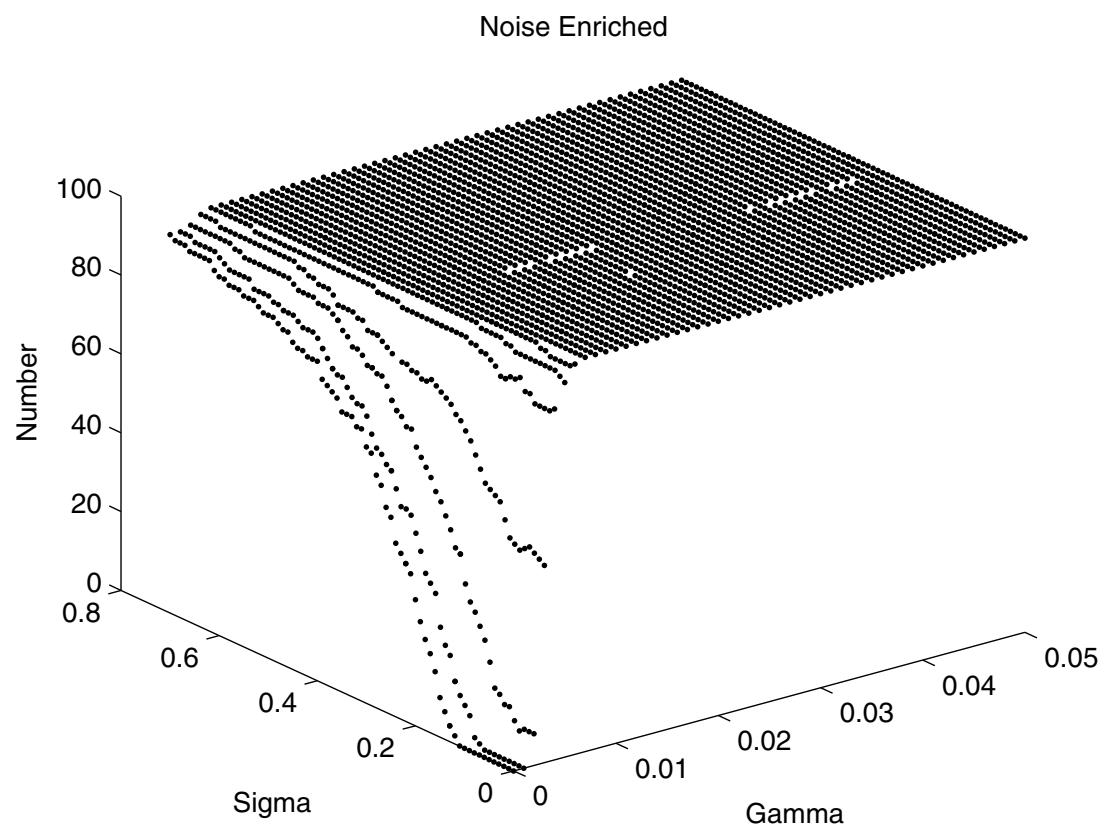

FIG. 13. The $(\sigma, \gamma)$ noise-enriched surface. 
model complexity increases), development of a theoretical and computational framework for sensitivity analysis of such models should prove most useful. Our preliminary efforts and results reported on here provide a sound foundation for further investigations in this direction.

As noted in 92, (2.1) fails to have smooth coefficients because $\left|A_{i}(\cdot)\right|$ changes discontinuously. Therefore, the solution is not a diffusion process in the usual sense. A smooth alternative to (2.1) is

$$
d \mathbf{C}_{i}(t)=\frac{\beta_{i}}{\sum_{i^{\prime} \neq i} e\left(i, i^{\prime}, t\right)} \sum_{i^{\prime} \neq i} e\left(i, i^{\prime}, t\right)\left[\mathbf{C}_{i^{\prime}}(t)-\mathbf{C}_{i}(t)\right] d t+\sigma d \mathbf{W}_{i}^{\mathbf{C}}(t) .
$$

In (5.1) the connectivities serve as weights. When combined with (2.2), (5.1) produces a smooth system in which - interestingly - edges or links have been replaced by smooth connectivities. More complete investigation of this system is a topic for future research.

Acknowledgements. This research was supported in part by the National Science Foundation under grant DMS-0437183 to the National Institute of Statistical Sciences (NISS), the U.S. Air Force Office of Scientific Research under grant AFOSR-FA9550-041-0220, and the National Science Foundation under grant DMS-0112069 to the Statistical and Applied Mathematical Sciences Institute (SAMSI). Any opinions, findings, and conclusions or recommendations expressed in this publication are those of the authors and do not necessarily reflect the views of the National Science Foundation. We wish to express our gratitude to Dr. Nathan Gibson for his assistance in parallel computing and to Dr. Michael Last for suggestions related to the regime definitions.

\section{REFERENCES}

[1] K. A. Bollen, Structural Equations with Latent Variables, Wiley Series in Probability and Mathematical Statistics, Wiley, New York, 1989. MR996025 (90k:62001)

[2] B. W. Bush, The TRAMSIMS simulation framework, 2001; Presented at the AgentBased Modeling Seminar, Los Alamos National Laboratory. Available on-line at www.lanl.gov/tools/media/realmetafiles/tsa/bbtransims.ram.

[3] K. Carley, Computational organizational science and organizational engineering, Simulation Modeling Practice and Theory 10:253-269, 2003.

[4] P. J. Carrington, J. Scott, and S. Wasserman, Models and Methods in Social Network Analysis, Cambridge University Press, New York, 2005.

[5] A. Chaturvedi, D. Dolk, and H. J. Sebastian, Agent-based simulation and model integration, 2004; Presented at the IFIP WG7.6 Workshop on Virtual Environment for Advanced Modeling (VEAM). Available on-line at web.nps.navy.mil/drdolk/ABS-Model-Integration.ppt.

[6] M. Dombroski, P. Fischbeck, and K. Carley, Estimating the shape of covert networks, in Proceedings of the 8th International Command and Control Research and Technology Symposium, National Defense War College, Washington, 2003.

[7] L. Festinger, The analysis of sociograms using matrix algebra, Human Relations 2:153-158, 1949.

[8] L. Festinger, A theory of social comparison processes, Human Relations 7:117-140, 1954.

[9] O. Frank and D. Strauss, Markov graphs, J. Amer. Statist. Assoc. 81:832-842, 1986. MR.860518 (88d:62163)

[10] T. C. Gard, Introduction to Stochastic Differential Equations, Marcel Dekker, Inc., New York, 1988. MR 917064 (88m:60157)

[11] G. R. Grummett and D. R. Stirzaker, Probability and Random Processes, $2^{\text {nd }}$ edition, Oxford University Press, Oxford, UK, 1992. MR.1199812 (93m:60002)

[12] M. Handcock, Progress in statistical modeling of drug user and sexual networks, Technical report, University of Washington, 2000 . 
[13] P. Hoff, A. Raftery, and M. Handcock, Latent space approaches to social network analysis, J. Amer. Statist. Assoc. 97:1090-1098, 2002. MR1951262

[14] J. Hollan, Adaptation in Natural and Artificial Systems, University of Michigan Press, Ann Arbor, MI, 1975. MR0441393 (55:14256)

[15] L. Katz, On the matrix analysis of sociometric data, Sociometry 10:233-241, 1947.

[16] L. Katz, Measurement of the tendency towards reciprocation of choice, Sociometry and the Science of Man 18:659-665, 1955.

[17] P. E. Kloeden, E. Platen, and H. Schurz, Numerical Solution of SDE Through Computer Experiments, Springer, New York, 1997. MR1260431 (95a:60084)

[18] J. L. Moreno, Who Shall Survive? Nervous and Mental Disease Publishing, Washington, 1934.

[19] K. Nowicki and T. Snijders, Estimation and prediction for stochastic block structures, J. Amer. Statist. Assoc., 96:1077-1087, 2001. MR 1947255

[20] T. Snijders, The SIENA Homepage, Available on-line at stat.gamma.rug.nl/snijders/index.html.

[21] T. Snijders, The statistical evaluation of social network dynamics, in Sociological Methodology, M. E. Sobel and M. P. Becker, eds., Basil Blackwell, Boston \& London, 2001, pp. 361-395.

[22] J. Von Neumann, Theory of Self-Reproducing Automata, University of Illinois Press, Urbana, IL, 1966.

[23] Y. J. Wang and G. Y. Wong, Stochastic blockmodels for directed graphs, J. Amer. Statist. Assoc., 82:8-19, 1987. MR883333

[24] S. Wasserman and K. Faust, Social Network Analysis: Methods and Applications, Cambridge University Press, New York, 1994.

[25] S. Wasserman and J. Galaskiewicz, Advances in Social Network Analysis: Research in the Social and Behavioral Sciences, Sage Publications, Thousands Oaks, CA, 1994.

[26] S. Wasserman and P. Pattison, Logit models and logistic regressions for social networks: I. An introduction to Markov graphs and $p^{*}$, Psychometrika, 61:401-425, 1996. MR 1424909 